\title{
Crystal-field induced dipoles in heteropolar crystals I: concept
}

\author{
Mario Birkholz \\ Ingenieurbüro für Solartechnik, Offenbacher Strasse 7, D-14197 Berlin, Germany \\ Received: 11 July 1994
}

\begin{abstract}
The electrostatic part of the internal energy of heteropolar crystals is largely assumed to be purely of the Coulomb or monopole type. Here, it is argued, ions in a crystal lattice may not only bear a net charge, but also higher electrostatic moments. This applies explicitly for dipole moments. Dipoles are assumed to occur only for ions on lattice sites where the point symmetry allows a non-vanishing crystal electric field to cause a polarization. Infinite lattice sums that account for the electrostatic interaction between point charges and dipoles are given, with the Madelung constant being the first of them in a more general Taylor expansion. An expression for the binding energy of heteropolar solids is hereby presented. The share due to induced dipoles is always negative if dipole-dipole interactions are neglected, i.e. it increases the strength of crystal binding. The concept, which is developed for crystals of arbitrary symmetry is explained on the basis of the examples (i) sphalerite ( $\mathrm{ZnS}$ ), (ii) pyrite $\left(\mathrm{FeS}_{2}\right)$, (iii) rutile $\left(\mathrm{TiO}_{2}\right)$, and (iv) orthorhombic $\mathrm{La}_{2} \mathrm{CuO}_{4}$.
\end{abstract}

PACS: $05.50 .+\mathrm{q} ;$ 61.50.Lt

\section{Introduction}

The internal energy of compounds crystallizing in the rock-salt or sphalerite structure can be accounted for by the ionic model that likens the solid to a gigantic molecule. The atoms within the molecule are assumed to ionize and interact mainly via long-range Coulomb potentials. Regarding the latter, when the interaction of one ion with the whole molecule is summed, it is found to yield specific values for certain crystallographic structures [1], which is well known as the Madelung constant. If repulsive forces between the electronic cores of neighboring ions are also included into the model, the internal energy of the solid can be computed. This figure is found to be in close agreement with an experimental value de- rived from the Born-Fajans-Haber thermodynamic cycle [2]. These early concepts of modern solid-state physics still play an important role in today's research: Madelung potentials are used in band structure calculations [3]. They are also linked to the elastic properties of the crystal as the bulk modulus [4], whereas the Born-Fajans-Haber thermodynamic cycle, for example, is used to estimate disorder energies [5].

Because the Madelung constant exclusively accounts for the interaction between electric monopoles, it is evident that for some solids the agreement between theoretical and experimental crystal energy might be better if higher order moments were included in the calculations. However, this point is relevant only for ions on positions, where symmetry considerations permit higher order moments. In rutile $\left(\mathrm{TiO}_{2}\right)$ and pyrite $\left(\mathrm{FeS}_{2}\right)$, which are investigated for solar cell applications $[6,7]$, chalcogen ions occupy positions where crystal electrical fields may occur, thereby causing the ions to become polarized. In $\mathrm{NaCl}$ and $\mathrm{ZnS}$, however, such fields cannot occur due to the point symmetry of the ion site $\left(O_{h}\right.$ and $\left.T_{d}\right)$. In their case only the sum of the potentials of other point charges yields a non-zero result, i.e. the Madelung constant. Therefore, it was not surprising that the crystal energy of rock-salt and sphalerite type compounds agreed well with the value of the thermodynamic cycle when calculated by a point charge ansatz [2] - whereas this was not the case for rutile [8] and pyrite [9]. For $\mathrm{FeS}_{2}$, however, it was shown that when dipole contributions are included for both the theoretical and experimental crystal energy, the difference becomes as small as $2 \%$ as compared with a deviation of $15 \%$ when only monopole interactions are added [9].

These results suggest the internal energy of heteropolar crystals can be fully accounted for if the electrostatic moments incorporated into the calculations are high enough. Alternatively, one might use a more general electrostatic model to describe binding energies in composite lattices instead of the usual ionic model. Such point multipole concepts have already been formulated [10-14]. They have been used to predict electrical field gradients 
measured by nuclear probes in some solids $[15,16]$. Because of the inclusion of dipoles and even higher moments, the theoretical results were in significantly better agreement with the experiment in these cases [17-21]. The importance of the approach has mainly been recognized by molecular physics, see $[22,23]$ and the references cited therein. However, most works were aimed at understanding the interaction between two or a small number of particles. Also for crystals composed of organic molecules, the significance of dipole moments for the cohesion of the solid was recognized [24] and is intensively used nowadays [25]. Recently, the interaction between adsorbed molecules on a crystal surface became a new field for the application of dipole theories [26]. It seems, however, that the question of induced dipoles in polar crystals has not gained as much attention as it deserves.

This work will present the general formulas of the second-order electrostatic lattice sums for ionic sites of arbitrary symmetry. For some special cases third and ever higher order moments will be of interest. However, this work will concentrate on dipole moments, because they are the most important corrections to the widely used first-order theory, and because they account already for many interesting physical phenomena. The presented formulas may be applied to any crystallographic structure. Much attention will be given to the point symmetry of the ionic sites, which determines the orientation of dipole moments and the number of independent components. The Born-Fajans-Haber thermodynamic cycle is reconsidered for crystals in which second-order moments may occur, and an expression for their binding energy will be given. The significance of ionic dipole moments for some physical properties of heteropolar crystals will be discussed in the second part of this work [27].

\section{Electrostatic lattice sums}

The so-called crystal energy or lattice energy $U_{\dot{L}}$ of a heteropolar solid is generally assumed to be composed of the electrostatic interaction $E_{\mathrm{e} 1}$, the repulsion between core electrons $E_{r}$, the van der Waals attraction $E_{v d W}$ and the energy due to thermal vibrations $E_{t v}$

$U_{L}=E_{\mathrm{el}}+E_{r}+E_{v d W}+E_{t v}$.

Because the last two terms are small compared to the first ones, the balance between electrostatic interaction and core-core repulsion is determinant for most ionic crystals. For an overhelming number of investigations, the electrostatic part was assumed to be fully accounted for by the Coulomb interaction (some hundred examples are compiled in [28]), being dependent as $1 / r_{i k}$ upon the distance between the $i$-th and $k$-th ion, $r_{i k}=r_{i}-r_{k}$. However, in general the interaction of the atom's charge distribution with other ones is given by a Taylor expansion [29] of which the first two terms are

$q_{i} \Phi-\left\langle\mathbf{p}_{i} \mid \mathbf{F}\right\rangle$ where $q_{i}$ and $p_{i}$ are the first and second electrical moment, i.e. charge and dipole of the $i$-th atom and $\Phi$ and $F$ being the potential and field at its site $r_{i}$ due to the other atoms. Therefore, second and perhaps even higher moments have to be considered to properly calculate the theoretical crystal energy. At least this approach should become important, whenever the crystal energy as estimated from the thermodynamic cycle deviates significantly from the theoretical one as calculated only with monopole interactions. If we restrict ourselves to the extension of $E_{\mathrm{e} 1}$ to second-order effects, the potentials and fields of monopoles and dipoles have to be inserted in (2). They are given by the infinite sums

$$
\begin{aligned}
& \Phi\left(\mathbf{r}_{i}\right)=\sum_{l, l \neq i} \frac{q_{l}}{4 \pi \varepsilon_{0} r_{i l}}+\sum_{l, l \neq i} \frac{\left\langle\mathbf{p}_{l} \mid \mathbf{r}_{i l}\right\rangle}{4 \pi \varepsilon_{0} r_{i l}^{3}} \\
& \mathbf{F}\left(\mathbf{r}_{i}\right)=\sum_{l, l \neq i} \frac{q_{l} \mathbf{r}_{i l}}{4 \pi \varepsilon_{0} r_{i l}^{3}}+\sum_{l, l \neq i} \frac{\left\langle\mathbf{p}_{l} \mid \mathbf{r}_{i l}\right\rangle \mathbf{r}_{i l}-r_{i l}^{2} \mathbf{p}_{l}}{4 \pi \varepsilon_{0} r_{i l}^{5}}
\end{aligned}
$$

that have to be extended over all lattice points (index $l$ ). SI units are used throughout the text with $\varepsilon_{0}$ being the permittivity of free space.

Concerning the dipole moments of the ions, some basic assumptions will be made in the following. First, it is assumed, that a dipole moment is associated with an ion only if a net crystal electrical field $F$ occurs at its lattice site. Second, the dipole $p$ is induced by the electric field, and both vectors are coupled by the dipole polarizability $\kappa, \mathbf{p}=4 \pi \varepsilon_{0} \kappa \mathbf{F}$. And third, the ionic polarizability is assumed to be a scalar quantity. The latter point is in contrast with some theories of dielectrics where it is found reliable to use a tensor polarizability to describe the macroscopic behaviour of anisotropic crystals. Fourth, as the ions are imagined to be point charges within the usual ionic model, the second-order moments will be assumed to be accounted for by point dipoles.

It must be emphasized that non-vanishing electric fields $F$ and dipole vectors $p$ do not occur at any arbitrary crystallographic position, but depend upon the point symmetry of the ionic site. For instance, when an ion is located at the intersection of three perpendicular mirror planes (point symmetry $D_{2 h}$ or $\mathrm{mmm}$ ), it is impossible for the surrounding charges to cause a net electrical field. Because for every charge $q_{k}$ at $r_{i k}$ there is another one of the same magnitude at $-r_{i k}$ that causes the fields of both to cancel at $r_{i}$, this also holds true for the field of dipoles, see (4). It is not the occurrence of the dipole alone, but also the number of its independent components that is determined by the symmetry of the lattice site.

In general, a dipole vector may have three different components, and each would serve as a new internal degree of freedom for the energy of the crystal. There are, however, symmetry conditions that constrain the number of independent components. For instance, a $n$ fold rotation axis passing through the ion forces the dipole to be oriented in this direction and to have only one component. A complete analysis $[22,30]$ leads to the compilation as presented in Table 1, where the number of independent dipole components is shown for 
Table 1. Number of independent dipole components allowed for ions in a crystal lattice versus point group symmetry of their lattice site. For 22 of 32 point groups crystal electrical fields and dipole moments induced by them do not occur because symmetry forbids them. The table can also be interpreted to give the well-known categorization of crystal classes by their number of independent polar axes

\begin{tabular}{lll}
\hline $\begin{array}{l}\text { Point groups } \\
\text { Schönfließ } \\
\text { notation }\end{array}$ & $\begin{array}{l}\text { International } \\
\text { notation }\end{array}$ & $\begin{array}{l}\text { number } \\
\text { of allowed } \\
\text { dipole } \\
\text { components }\end{array}$ \\
\hline & & \\
\hline$C_{1}$ & 1 & 3 \\
$C_{s}\left(C_{1 h}\right)$ & $\mathrm{m}$ & 2 \\
$C_{2}, C_{3}, C_{4}, C_{6}$ & $2,3,4,6$ & 1 \\
$C_{2 v}, C_{3 v}, C_{4 v}, C_{6 v}$ & $\mathrm{~mm} 2,3 \mathrm{~m}, 4 \mathrm{~mm}, 6 \mathrm{~mm}$ & 1 \\
$S_{2}\left(C_{i}\right), S_{4}, S_{6}\left(C_{3 i}\right)$ & $\overline{1}, \overline{4}, \overline{3}$ & 0 \\
$C_{2 h}, C_{3 h}, C_{4 h}, C_{6 h}$ & $2 / \mathrm{m}, \overline{6}, 4 / \mathrm{m}, 6 / \mathrm{m}$ & 0 \\
$D_{2}(V), D_{3}, D_{4}, D_{6}$ & $222,32,422,622$ & 0 \\
$D_{2 h}\left(V_{h}\right), D_{3 h}, D_{4 h}, D_{6 h}$ & $\mathrm{mmm}, \overline{6} \mathrm{~m} 2,4 / \mathrm{mmm}$, & 0 \\
$D_{2 d}\left(V_{d}\right), D_{3 d}$ & $6 / \mathrm{mmm}$ & \\
$O_{h}, O, T_{h}, T_{d}, T$ & $\overline{4} 2 \mathrm{~m}, \overline{3} \mathrm{~m}$ & 0 \\
\hline & $\mathrm{m} \overline{3} \mathrm{~m}, 432, \mathrm{~m} \overline{3}, \overline{4} 3 \mathrm{~m}, 23$ & 0 \\
\hline
\end{tabular}

all 32 crystallographic point groups, as given in Schönflie $B$ and international notation. It is evident, that it is the same table as for the number of polar axes of pyroelectric crystals (see [4], the discussion of this point is postponed to the second part of this work). It can be seen that, for more than half of all point groups, dipole moments are forbidden due to symmetry reasons. In most cases, when a net electric field occurs, the dipole has only one independent component. For point group $C_{1 h}$ (or m) with one mirror plane as a symmetry element two independent vectors define this plane, whereas $C_{1}$ (or 1) allows for three components. The orientation of dipole vectors is visualized for the two examples of point group $C_{4 v}$ (or $4 \mathrm{~mm}$ ) and $C_{1 h}$ in Fig. 1 .

For the calculation of the Madelung constant a charge $q_{i}=z_{i} e$ is assigned to every sort of ion in the lattice, e.g. $z_{\mathrm{Cl}}=-1$ in $\mathrm{NaCl}$. The choice of the charge's

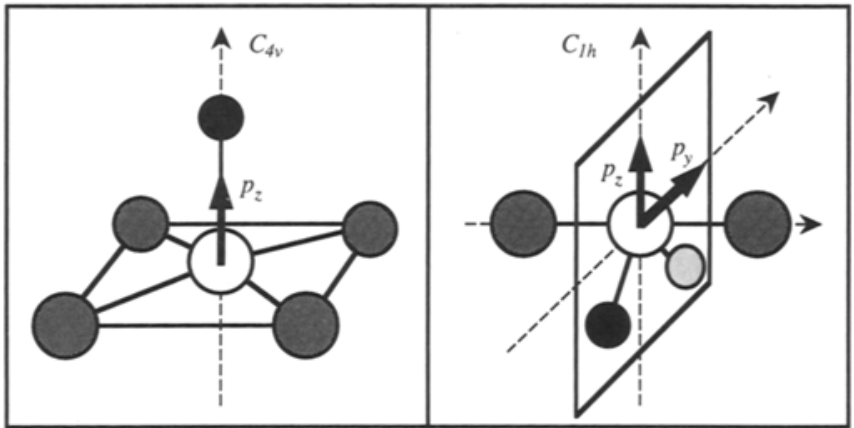

Fig. 1. Schematic drawing of point group symmetry $C_{4 v}$ and $C_{1 h}$ (Schönfließ notation). One dipole component $p_{z}$ is allowed for the central atom in a $C_{4 v}$ coordination, which has to be directed along the axis of rotation. For $C_{1 h}$, however, there are two degrees of freedom for the dipole vector, $p_{y}$ and $p_{z}$, which must lie within the mirror plane magnitude is determined by the next rare gas or closed electron shell. However, it is not possible to specify a certain value a priori for the strength of the second moment. Instead, the dipole's strength will have to be calculated, which can be done with a system of equations to be given later. One must therefore evaluate the potentials and fields of all dipoles without knowing their strength, see the second term in (3) and (4). The problem can be bypassed by introducing dipole unit vectors $\mathbf{n}$ with $\mathbf{p}=p \mathbf{n}$. The orientation of $\mathbf{n}$ has to be chosen in accordance with the point symmetry as discussed above. The unit vectors are of the form $(100),(110) / \sqrt{2},(111) / \sqrt{3}$ etc. depending on whether the axis of rotation is parallel to a cell edge, along a face diagonal or a body diagonal. If there is more than one independent dipole component allowed, two or three unit vectors must be assigned to that ion.

For the set of ions in a crystallographic unit cell, it will be useful to first assign dipole unit vectors to the ions of the asymmetric unit. If their positions are abbreviated by $r_{i}$, the other ions of the unit cell, on $r_{i}^{\prime}$, are generated by symmetry operations, $r_{i}^{\prime}=S r_{i}+t$, that consist of a rotation $S$ and a translation $t$. Their dipole vectors are oriented along $n_{i}^{\prime}$, which follows from the rotation $n_{i}^{\prime}=S n_{i}$, whereas the translation does not apply on $n_{i}$.

The calculation of the electrostatic interaction of a heteropolar crystal proceeds as follows. Assuming that the unit cell contains $N$ sorts of ions with charges $q_{i}$, and $M$ different dipole moments, $p_{j}$. If there are $Z$ formula units per unit cell, and if $K_{i}$ and $L_{j}$ are the site occupancies of the $i$-th ion and the $j$-th dipole, (2) may be rewritten as the electrostatic interaction energy per formula unit of the compound

$E_{\mathrm{el}}=\sum_{i=1}^{N} \frac{K_{i}}{2 Z} q_{i} \Phi\left(r_{i}\right)-\sum_{j=1}^{M} \frac{L_{j}}{2 Z}\left\langle\mathbf{p}_{j} \mid \mathbf{F}\left(r_{j}\right)\right\rangle$

with the factor $\frac{1}{2}$ being introduced to avoid double summation of energies. It should be noticed that indices $i$ and $j$ may numerate the ions within the unit cell in a totally different manner, depending on whether a dipole moment is associated with an ion and on the number of its independent components.

In general, a crystal belonging to the tricline system has three different unit cell edges $a, b, c$ and three different angles between them: $\alpha=\Varangle(\mathbf{b}, \mathbf{c}), \beta=\Varangle(\mathbf{c}, \mathbf{a})$, $\gamma=\Varangle(\mathbf{a}, \mathbf{b})$. The volume of the unit cell $V$ is that of the parallelepiped

$$
\begin{aligned}
V= & a b c\left(1-\cos ^{2} \alpha-\cos ^{2} \beta-\cos ^{2} \gamma\right. \\
& +2 \cos \alpha \cos \beta \cos \gamma)^{1 / 2} .
\end{aligned}
$$

The cube root of the volume $w=\sqrt[3]{V}$ will be used in the following for the normalisation of distances $\rho_{i k}$ $=r_{i k} / w$. Dipole moments will be normalized, $\mu=p / e w$, with respect to $w$ and the elementary charge $e$. It is useful to introduce the covariant metric tensor $g_{\lambda \nu}$, the components of which are the direct products of the unit cell vectors $\mathbf{a}, \mathbf{b}$ and $\mathbf{c}$ 
$g_{\lambda_{v}}=\left(\begin{array}{ccc}a^{2} & a b \cdot \cos \gamma & a c \cdot \cos \beta \\ b a \cdot \cos \gamma & b^{2} & b c \cdot \cos \alpha \\ c a \cdot \cos \beta & c b \cdot \cos \alpha & c^{2}\end{array}\right)$

With its help, the inner product of two vectors - the contravariant components of which are given in fractions of $a, b$ and $c$ - is calculated, for instance for $\mathbf{n}$ and $\rho$, as follows

$$
\langle\boldsymbol{\rho} \mid \mathbf{n}\rangle=\sum_{\lambda, v=1}^{3} \rho^{\lambda} g_{\lambda v} n^{v} .
$$

Finally, one is in a position to formulate the electrostatic interaction up to second order in a three-dimensional crystal of general symmetry. With the above definitions (5) becomes

$$
\begin{aligned}
E_{\mathrm{el}}= & \frac{E_{0}}{2}\left[\sum_{i=1}^{N} \frac{K_{i}}{Z} z_{i}\left(\alpha_{i}^{m}+\sum_{j=1}^{M} \mu_{j} \alpha_{i j}^{d}\right)\right. \\
& \left.-\sum_{j=1}^{M} \frac{L_{j}}{Z} \mu_{j}\left(\beta_{j}^{m}+\sum_{k=1}^{M} \mu_{k} \beta_{j k}^{d}\right)\right] .
\end{aligned}
$$

The $\alpha$ and $\beta$ terms are infinite lattice sums that yield definite values for every special crystallographic structure. They will sometimes be named electrostatic lattice coefficients in the following. $\alpha$ terms account for the interaction of charges with potentials, whereas $\beta$ coefficients describe the interaction between dipoles and crystal electrical fields. The superscripts $m$ and $d$ specify whether the interaction is with monopoles or dipoles. $\alpha_{i}^{m}$ then is the familiar Madelung constant of the $i$-th ion. It has been mentioned that the actual values of dipole strength $\mu_{j}$ cannot as easily assigned to the ions as can the charges $q_{i}$. This causes $\alpha^{d}$ of the $i$-th ion to split into $M$ terms, each accounting for the interaction of $q_{i}$ with the potential of the dipoles of the $j$-th sort. $M$ different $\alpha_{i j}^{d}$ then are obtained. The same holds true for the set of $\beta^{d}$ sums.

The electrostatic interaction energy within the solid, $E_{\mathrm{el}}$, scales in units of $E_{0}=e^{2} / 4 \pi \varepsilon_{0} w=2 R y d a_{0} / w$, which appears to be the "natural" energy unit of the system. Since Bohr's radius is roughly half an Ångstrom, while $w$ in most cases ranges from $4-10 \AA, E_{0}$ will usually be of the order of approximately $2 \mathrm{eV}$. It should be remembered that $\mu$ stands for the normalized dipole strength. After reinserting $\mu=p / e w$ into (9), the single terms are seen to be proportional to $1 / w, 1 / w^{2}$ and $1 / w^{3}$, so the exponents of each specific electrostatic interaction can be recognized $(m m, m d, d m, d d)$. The first electrostatic lattice sums for the Taylor expansion of $E_{\mathrm{el}}$ are explicitly as follows

$$
\begin{aligned}
& \alpha_{i}^{m}=\sum_{l, l \neq i} \frac{z_{l}}{\rho_{i l}} \\
& \alpha_{i j}^{d}=\sum_{l, l \neq i} \frac{\left\langle\boldsymbol{\rho}_{i l} \mid \mathbf{n}_{l}(j)\right\rangle}{\rho_{i l}^{3}} \\
& \beta_{j}^{m}=\left\langle\mathbf{n}_{j} \mid \sum_{l, l \neq j} \frac{z_{l} \boldsymbol{\rho}_{j l}}{\rho_{j l}^{3}}\right\rangle
\end{aligned}
$$

$\beta_{j k}^{d}=\left\langle\mathbf{n}_{j} \mid \sum_{l, l \neq j} \frac{3\left\langle\mathbf{n}_{l}(k) \mid \boldsymbol{\rho}_{j l}\right\rangle \boldsymbol{\rho}_{j l}-\rho_{j l}^{2} \mathbf{n}_{l}(k)}{\rho_{j l}^{5}}\right\rangle$

with direct products to be taken in accordance with (8) and $n_{l}(k)$ serves as $l$-th unit vector of the $k$-th type of dipoles. Because $\beta^{d}$ coefficients act on the third power of $1 / w$, it would perhaps be more adequate to incorporate them into a theory that can account for the full third order of electrostatic interaction. As is the Madelung constant, all electrostatic lattice coefficients are pure geometrical sums of the crystal lattice that can be calculated when all structural parameters have been determined (in contrast to [9] the definitions in $(10 \mathrm{a}-\mathrm{d})$ have slightly been changed, i.e. the factor $\frac{1}{2}$ is missing within the sums, and $z_{i}$ has been deleted in $\alpha^{m}$ and $\alpha^{d}$ ).

As has been mentioned, this work is concerned with crystals having $N$ different types of ions with charges $e z_{j}(j=1, \ldots, N)$. It can be shown that a decomposition of Madelung's constant into single parts, each caused by a different ion

$\alpha_{i}^{m} \neq \sum_{j=1}^{N} z_{j} \sum_{l, l \neq i} \frac{1}{\rho_{i l}(j)}=\sum_{j=1}^{N} z_{j} A_{i j} \quad A_{i j} \neq 0 \forall i j$,

is impossible, because the partial sums diverge. Only by adding the charges weighted by the inverse distance $1 / \rho$ in the "right" order, a convergent sum is obtained. Mathematically speaking, this is caused by the divergence of the harmonic sum. However, for the $\beta^{m}$ sums according to formula $(10 \mathrm{c})$, the charges are multiplied by the inverse square of the distance $1 / \rho^{2}$ before being added. One may ask whether a separation into sums over different ions is possible. The answer to this question is that such a decomposition can be obtained, and it reads like

$\beta_{j}^{m}=-\sum_{i=1}^{N} \frac{K_{i}}{L_{j}} z_{i} \alpha_{i j}^{d}$

where $L_{j}$ and $K_{i}$ describe the frequencies of the $j$-dipole and the $i$-charge in the unit cell. The physical meaning of this relation simply is that the interaction of a set of charges with the potential of dipoles equals the interaction of dipoles with the field of charges. It was first determined by Rudert [31] to hold for $\beta^{m}$ of the sulfur ions in $\mathrm{FeS}_{2}$. Eq. (12) will help considerably in simplifying some of the forthcoming expressions.

The strengths of the dipoles may be regarded as a degree of freedom of the system. They can be determined with the help of the $\beta$ type sums. Assuming $\mathbf{p}=4 \pi \varepsilon_{0} \kappa \mathbf{F}$, with $\kappa$ being the dipole polarizability of the ion, there are two ways to calculate the set of $p_{j}$. First, it may be assumed, that dipole moments are mainly induced by the fields of monopoles $F^{m}$. These moments will be abbreviated by $\phi_{j}$ in their normalized form $p_{j} / e w$ and it is obtained

$\phi_{j}=\frac{\kappa_{j}}{V} \beta_{j}^{m}$.

Alternatively, one may assume that moreover the dipoles' electric field $F^{d}$ will modify dipole moments recur- 
sively. This set of dipoles is symbolised by $\mu_{j}$ to distinguish them from the above set. This results in

$\mu_{j}=\frac{4 \pi \varepsilon_{0} \kappa_{j}}{w e}\left(F_{j}^{m}+\sum_{k=1}^{M} F_{j k}^{d}\right)=\frac{\kappa_{j}}{V}\left(\beta_{j}^{m}+\sum_{k=1}^{M} \mu_{k} \beta_{j k}^{d}\right)$,

which is solved to yield a linear system of equations for the set of the $\mu_{j}$

$\sum_{k=1}^{M}\left(\delta_{j k} \frac{V}{\kappa_{j}}-\beta_{j k}^{d}\right) \mu_{k}=\beta_{j}^{m}$

with $\delta_{j k}$ being Kronecker's symbol. The left-hand side of $(15 \mathrm{a})$ is seen to be the product of a matrix $B_{j k}$ and the vector $\mu_{k}$. If both sides are multiplied by the inverse of $B_{j k}$, the set of equations

$\mu_{k}=\sum_{j=1}^{M} B_{k j}^{-1} \beta_{j}^{m} \quad B_{j k}=\delta_{j k} \frac{V}{\kappa_{j}}-\beta_{j k}^{d}$

is obtained. This becomes equal to (13) for a vanishing dipole-dipole interaction, or $\beta_{j k}^{d}=0$. The dipole strength then is sufficiently well approximated by the set of the $\phi_{j}$. By using (12) (15b) is reformulated to become

$\mu_{k}=-\sum_{j=1}^{M} \sum_{i=1}^{N} \frac{K_{i}}{L_{j}} z_{i} \alpha_{i j}^{d} B_{k j}^{-1}$.

This formula shows the dipole strengths to depend on the ionic polarisabilites $\kappa_{j}$, on the distribution of charges over the different lattice sites and - via $\alpha^{d}$ and $\beta^{d}$ sums - on the geometry of the lattice. While the dipoles scale lineary with the charges $z_{i}$ the dependency on the set of $\kappa_{j}$ is more complicated, because the latter enter via a rational fraction of specific polarisabilities $\kappa_{j} / V$ through the matrix $B_{k j}$. Only for negligible dipole-dipole interaction the strength of the dipoles becomes a linear function of the polarizabilities.

So far, the crystal has been assumed to be static and the ions or dipoles were imagined to reside on fixed positions. However, at finite temperatures, the lattice constitutents will move with respect to each other in an irregular manner due to thermal vibrations. The dipole strength of an ion $p$ at a dipole-allowed lattice site will then become a time-dependent function, $p(t)$, as is its position. We will assume, however, that its mean value, $\bar{p}$, is not equal zero. It is this special $\bar{p}$ that is accounted for by the presented formulas.

Finally, all terms of (9) have been determined. The electrostatic interaction in a heteropolar crystal can be calculated to second order, if the structural parameters and dipole polarizabilities of the ions are known.

\section{The Born-Fajans-Haber thermodynamic cycle and the binding energy of crystals}

The crystal energy or lattice energy $U_{L}$ as given in (1) should be released, if the gaseous ions are moved from infinity to form the crystal. According to the Born-Fa-

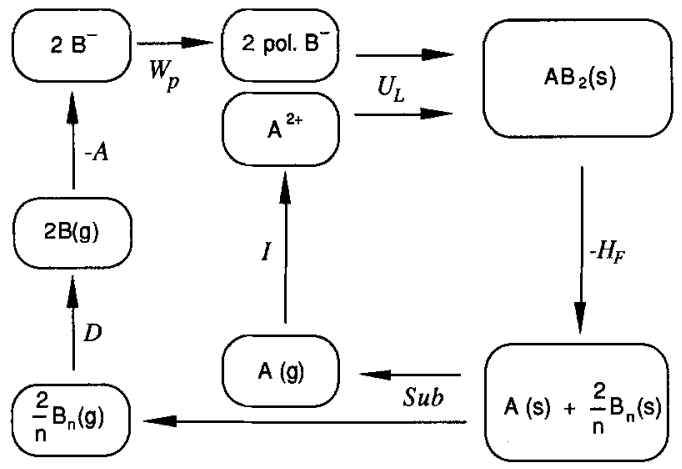

Fig. 2. Born-Fajans-Haber thermodynamic cycle of a model compound $A B_{2}$ with the usual process energies: negative heat of formation, $-H_{f}$, sublimation, $S u b$, dissociation, $D$, ionization, $I$, electron affinity, $A$, and the lattice energy, $U_{L} . B$ ions are assumed to be more electronegative and polarizable than $A$ ions, and they occupy crystallographic positions where second-order moments are induced. It is argued that a new step to polarize the $B$ ions has to be inserted within the cycle to account for the dipole moments in the lattice (polarization work $W_{p}$ ). It occurs after the acceptance of electrons by $B$ atoms. A new lattice energy $U_{L}=W_{\mathrm{BFH}}-W_{p}$ results instead of the usual Born-Fajans-Haber energy $W_{\mathrm{BFH}}$

jans-Haber thermodynamic cycle, the theoretically calculated lattice energy $U_{L, \text { th }}$ may be compared with an experimentally derived quantity, see Fig. 2. This cycle of chemical and physical reactions starts by converting the crystal to its elements, which is accounted for by the negative heat of formation $\left(-H_{f}\right)$. The elements are then assumed to be sublimized to the gas phase $(S u b)$, dissociated $(D)$ and ionizated to their proper charges (ionziation potential $I$ and negative electron affinity $-A$ ). The sequence of processes becomes a closed cycle if the ions are now brought together to form a solid. The lattice energy according to Born-Fajans-Haber becomes $U_{\mathrm{BFH}}=H_{f}-S u b-D-I+A$. If the theoretical ansatz accounts in the right manner for the lattice energy, $U_{L, \text { th }}$ and $U_{\mathrm{BFH}}$ should be equal.

The introduction of higher electrostatic moments has two important consequences. The first one was outlined in the foregoing section by inserting second-order energy terms within (9). This causes the theoretical lattice energy $U_{L, \text { th }}$ to become modified. The second consequence concerns the thermodynamic cycle itself. The Born-FajansHaber cycle adds the energy used to ionizate the atoms ( $I$ and $A$ ), so that they bear a first-order electrostatic moment. It was argued that dipole moments should be accounted for if the ions are situated on dipole-allowed lattice sites. Consequently, the energy used to polarize the ions should also enter the thermodynamic cycle. It, therefore, seems to be reasonable, to include a term $W_{p}$ for the polarization work within the cycle. Its strength follows from the fact that the energy to induce a dipole $p$ by an electric field $F$ is as large as $p F / 2$, which can be expressed for one formula unit of the compound as

$W_{p}=\sum_{j=1}^{M} \frac{L_{j}}{2 Z}\left\langle\mathbf{p}_{j} \mid \mathbf{F}\left(r_{j}\right)\right\rangle$

This step would occur within the cycle after the ionization of the atoms, as is shown in Fig. 2. Instead of $U_{\mathrm{BFH}}$ 
another lattice energy $U_{L}=U_{\mathrm{BFH}}-W_{p}$ must be evaluated. Because $U_{\mathrm{BFH}}$ is less than zero, and because $W_{p}$ is positive, the new lattice energy is apparently more negative than $U_{\mathrm{BFH}}$. Therefore, it is concluded that the Born-Fajans-Haber energy underestimates the lattice energy of crystals with ions on dipole-allowed positions.

It is interesting to realize, how the sum for the electrostatic interaction and the work of polarization looks like, if the relations are inserted as derived above. From (5), (9) and (17) one obtains

$$
E_{\mathrm{el}}+W_{p}=\frac{E_{0}}{2} \sum_{i=1}^{N} \frac{K_{i}}{Z} z_{i}\left(\alpha_{i}^{m}+\sum_{j=1}^{M} \mu_{j} \alpha_{i j}^{d}\right)
$$

The polarization work and the energy due to the interaction of dipoles with electrical fields have cancelled each other. However, the dipoles remain included due to the interaction of their electric potentials with monopoles. If the decomposition of the $\beta^{m}$ coefficient according to (12) is inserted recursively,

$E_{\mathrm{e} 1}+W_{p}=\frac{E_{0}}{2}\left(\sum_{i=1}^{N} \frac{K_{i}}{Z} z_{i} \alpha_{i}^{m}-\sum_{j=1}^{M} \frac{L_{j}}{Z} \mu_{j} \beta_{j}^{m}\right)$

one will find that the double sum of $\alpha^{d}$ coefficients reduces to a single one and we are left with two separate sums, one over all monopoles and the second over all dipoles. Again, the dipole-dipole interaction may be assumed to be small, $\beta^{d} \rightarrow 0$ or equivalently $\mu_{j} \rightarrow \phi_{j}$, and the expression finally reads like

$E_{\mathrm{el}}+W_{p} \cong \frac{E_{0}}{Z}\left(\sum_{i=1}^{N} \frac{K_{i}}{2} z_{i} \alpha_{i}^{m}-w^{3} \sum_{j=1}^{M} L_{j} \frac{\left(\phi_{j}\right)^{2}}{2 \kappa_{j}}\right)$.

The second sum has now taken the usual form of the energy of dipoles as indced in an external electrical field. Its significance has been fully realized in molecular science [23], where it is named induction energy or polarization energy, $E_{\mathrm{pol}}$.

One is now in the position to obtain an expression for the binding energy $E_{B}$ of a heteropolar solid, which is correct up to second-order electrostatics. As usual, $E_{B}$ is assumed to be the difference between the energies of bound atoms and non-interacting particles. For one formula unit of the solid, $E_{B}$ is seen from Fig. 2 to be the sum of all ionization energies $I$, electron affinities $A$, polarization work $W_{p}$ plus the crystal energy $U_{L}$, which yields with the help of (1), (20) and (13)

$$
\begin{aligned}
E_{B} \cong & \sum_{i=1}^{N} \frac{K_{i}}{Z}\left(I_{i}-A_{i}+\frac{E_{0}}{2} z_{i} \alpha_{i}^{m}\right) \\
& -\frac{E_{0}}{2} \sum_{j=1}^{M} \frac{L_{j}}{Z} \frac{\kappa_{j}}{w^{3}}\left(\beta_{j}^{m}\right)^{2}+E_{r}+E_{v d W}+E_{t v}
\end{aligned}
$$

The binding energy due to dipoles as induced by the crystal electric fields can be seen to be always negative if dipole-dipole interactions may be neglected, i.e. they increase the crystal binding. Moreover, the equation may also provide the binding energy $E_{B, i}$ for the $i$-th sort of ions in the crystal, because all terms can be attributed to a certain ion. This may be of importance when comparing the binding energy of the same sort of ion in different solids or the binding energy of different ions in the same solid.

It can also be seen that $E_{B}$ depends greatly upon the choice of $z_{i}$ that are assigned to the ions on the lattice. The $z_{i}$ may even be regarded as free parameters of the system, the minimum energy of which is determined by their proper choice according to

$\left.\frac{\partial E_{B}}{\partial z_{i}}\right|_{\Sigma K_{i} z_{i}=0}=0$

with the condition describing the charge neutrality of the crystal. This approach offers a quantitative solution to the problem of assigning charges $z_{i}$ to the ions by minimizing the system's energy. Instead of arguing on the basis of electron configurations of noble gases, (22) provides a quantitative recipe to determine a reliable choice. Because only discrete values are allowed for the set of $z_{i}$, this equation will lead to a comparison of different models of charge assignments. This will be explained in example one in the following section.

\section{Examples}

\section{Cubic ZnS}

Sphalerite type structure. Space group $T_{d}^{2}$, components of the metric tensor $g_{\lambda v}=a^{2} \delta_{\lambda v}$, unit length $w=a=5.42 \AA$, formula units in the (non-primitive) unit cell $Z=4$, sorts of ions $N=2$. The point symmetry of the ion's site is $T_{d}$. According to Table 1 the crystal electric field vanishes at ionic sites of such symmetry, and no dipole moment can be induced. Therefore, it applies for the number of dipoles in the unit cell, $M=0$. The dipole unit vectors $\mathbf{n}_{i}$ must be set to zero, causing all second-order electrostatic lattice sums to vanish, $\beta^{m}$ $=\beta^{d}=0$. Only the potential of other point charges yields a finite value that is the same for both ions. This value is the well-known Madelung constant of the ZnS-structure: $\alpha_{\mathrm{Zn}}^{m}=-\alpha_{\mathrm{S}}^{m}=-3.7829$ (normally $\alpha^{m}$ is given according to the distance to the nearest neighbour, which is obtained by multiplying the value by $\sqrt{3} / 4$ ). The electrostatic interaction until to second-order effects in sphalerite-type compounds is therefore fully accounted for by the first sum of (9). (22) can be applied to find the proper charges of the ions. Two different models will be investigated with $E_{B}^{1}$ and $E_{B}^{2}$ accounting for their different binding energies. The ions in model (i) are assumed to be ionizated onefold $\left(\mathrm{Zn}^{+} \mathrm{S}^{-}\right)$and twofold in model (ii) $\mathrm{Zn}^{2+} \mathrm{S}^{2-}$. There is good reason to suppose that energies due to repulsion, thermal vibrations and van der Waals interaction are equal in both models, so the difference in binding energies $\Delta E_{B}$ is solely due to $\Delta E_{\mathrm{el}}-\Delta I+\Delta A$. If first and second ionisation energies of $\mathrm{Zn}\left(I_{1}=9.39\right.$, $I_{2}=17.96 \mathrm{eV}$ according to [32]) and electron affinities of sulphur $\left(A_{1}=2.07, A_{2}=-5.51 \mathrm{eV},[33]\right)$ are used, $E_{B}^{2}-E_{B}^{1}$ is found to be $3 E_{0} \alpha^{m} / 2+I_{2}-A_{2}=-6.75 \mathrm{eV}$. Si- 
milary, the difference $E_{B}^{2}-E_{B}^{3}$ is found to be less than zero. These results predict that the binding energy of the $\mathrm{Zn}^{2+} \mathrm{S}^{2-}$-configuration will be smaller than that of one- and threefold charged ions. This substantiates this generally used assignment of formal valencies.

\section{2. $\mathrm{Cubic} \mathrm{FeS}_{2}$}

Pyrite-type structure. Space group $T_{h}^{6}$. The ions, their charges and point symmetries are described by the triples. $\left(\mathrm{Fe},+2, C_{3 i}\right) \quad$ and $\quad\left(\mathrm{S},-1, C_{3}\right) . \quad g_{\lambda v}=a^{2} \delta_{\lambda v}$, $w=a=5.41870(6) \AA$ [34], $Z=4, N=2$. Dipole moments are only allowed for sulfur ions, $M=1$. Their unit vectors are oriented along the body diagonals of the cube and they are obtained from the set of all combinations of $( \pm 1, \pm 1, \pm 1) / \sqrt{3}$ (see Fig. 3). Two Madelung coefficients and two $\alpha^{d}$ sums occur, each for one type of ion. $\beta^{m}$ and $\beta^{d}$ differ from zero only for sulfur ions, because electrical fields vanish at the $\mathrm{Fe}$ site. The electrostatic interaction between the ions is therefore characterized by five different electrostatic lattice sums. Their values are given - together with S-dipole moment and $E_{\text {el }}$ in [9]. The difference between theoretical and experimental lattice energy $U_{L}$, was found to be as large as $15 \%$ when dipole contributions were not taken into account. It decreased to $2 \%$, if dipole moments were included into the electrostatic interaction and within the thermodynamic cycle. The effect of dipole-dipole interaction was found to contribute only $14 \%$ of the total dipole strength being mainly induced by the point charges of the crystal lattice.

\section{Tetragonal $\mathrm{TiO}_{2}$}

Rutile-type structure. Space group $D_{4 h}^{14}$. $\left(\mathrm{Ti},+4, D_{2 h}\right)$, $\left(\mathrm{O},-2, C_{2 v}\right), g_{1 v}=a^{2} \delta_{1 v}, g_{2 v}=a^{2} \delta_{2 v}, g_{3 v}=c^{2} \delta_{3 v}$. w $=\sqrt[3]{a^{2} c} . Z=2, N=2$. Dipole vectors are forbidden for Ti ions and allowed for $\mathrm{O}$ ions, $M=1$, with unit vectors directed along the face diagonal of the basal plane, i.e. combinations of $( \pm 1, \pm 1,0) / \sqrt{2}$. The number of first and second-order electrostatic lattice coefficients is the same as in pyrite. The significance of dipole lattice sums for the calculation of the dielectric constant had already been recognized by Parker [35], but she rejected the occurrence of crystal field induced dipoles. Madelung constants were presented for varying $c / a$ ratios and oxygen site positional parameters by Baur [8]. $\alpha^{d}, \beta^{m}$ and $\beta^{d}$ sums can be extracted from Bertaut [14] and Kingsbury [36]. The latter also gave a solution according to (14) for $p(\mathrm{O})$ in $\mathrm{TiO}_{2}$.

\section{Orthorhombic $\mathrm{La}_{2} \mathrm{CuO}_{4}$}

$\mathrm{K}_{2} \mathrm{NiO}_{4}$-type structure [37]. Space group $D_{2 h}^{18}$. $(\mathrm{Cu}$, $\left.+2, C_{2 h}\right),\left(\mathrm{La},+3, C_{1 h}\right),\left(\mathrm{O} 1,-2, C_{2}\right),\left(\mathrm{O} 2,-2, C_{1 h}\right)$, $g_{1 v}=a^{2} \delta_{1 v}, g_{2 v}=b^{2} \delta_{2 v}, g_{3 v}=c^{2} \delta_{3 v} . w=\sqrt[3]{a b c} . Z=4$.

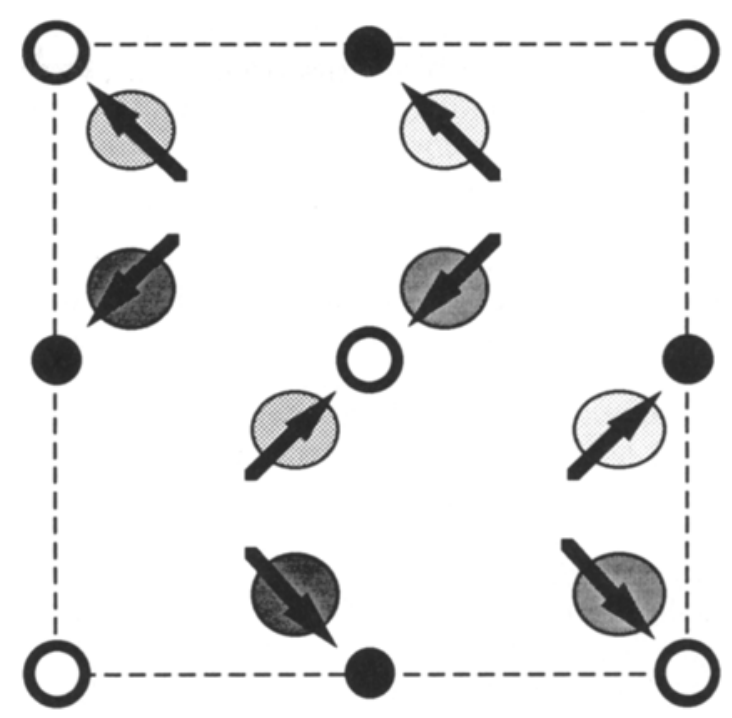

Fig. 3. Projection of the crystallographic unit cell of the cubic pyrite structure. Iron atoms build up an FCC sublattice and are indicated at the heights $0,1 / 2$ and 1 by small white and black circles. The sulfur atoms are differently shaded according to their different heights. The arrows indicate the dipole vectors that are oriented along the body diagonals of the cube

Dipole moments are forbidden for $\mathrm{Cu}$-ions, but allowed for all others. Unit vectors for O1 ions have one component. Because of the $C_{1 h}$ symmetry there arise two independent dipole moments for both the $\mathrm{O} 2$ site and the La site, respectively. One is directed along the $b$-axis and the other along the $c$-axis. It seems that this point has not been realized in the literature on the compound. The electrostatic interaction in the crystal is due to four charges and five second-order moments, $N=4, M=5$. Therefore, 49 different coefficients must be evaluated in accordance with second-order theory: $4 \alpha^{m}, 4 \times 5 \alpha^{d}$ and $5 \times 5 \beta^{d}$. Their values and the resulting dipole strengths for the $\mathrm{La}, \mathrm{O} 1$ and $\mathrm{O} 2$ ions are given for $T \approx 15 \mathrm{~K}$ in [38]. It is well known that $\mathrm{La}_{2} \mathrm{CuO}_{4}$ becomes superconducting when $\mathrm{La}$ ions are substituted by $\mathrm{Ba}$ or $\mathrm{Sr}$ ions $[39,40]$. To find the distribution of charges and the valencies of the ions by applying (22), the first and secondorder electrostatic lattice sums have been calculated for $\mathrm{La}_{2-x} \mathrm{Sr}_{x} \mathrm{CuO}_{4}$ in [38].

\section{Conclusions}

Symmetry conditions were presented for the occurrence of dipole moments induced by crystal fields, and geometric lattice sums that account for second-order electrostatic effects in crystals were given [41]. An expression for the binding energy that can be separated into the individual atomic fractions that consitute the solid was also presented. Precise structural information on the crystal under investigation is neccessary for the application of the concept. The most important restriction is the lack of precision of available data on electron affinities and polarizabilities. The second electron affinity of many elements, for instance, can be given only with poor precision 
in the range of $10 \%$, because these electronic configurations are unstable if not subjected to a crystal field. In the case of ionic polarizabilities, however, accurate methods that make use of the local density approximation (LDA) are now being developed, see [42] for a recent review.

The concept can naturally be extended for even higher-order electrostatic theory. For instance, electric field gradients of the ions that are allowed at special lattice sites may be assumed to induce quadrupole moments, just as the electrical field excites the dipole moment. This approach will lead to further electrostatic lattice constants of the $\gamma$-type, indicating that they account for field gradients, and to new superscripts $q$ symbolising third order electrical moments $\left(\alpha^{q}, \beta^{q}, \gamma^{m}, \gamma^{d}, \gamma^{q}\right.$ etc.). The most relevant application of $\gamma$-type coefficients is in the prediction of electric field gradients at certain lattice sites. They will probably be of minor importance for the crystal energy, because the strength of electrostatic interaction energy is coupled to the radial exponent by which it acts. Whereas the $1 / r$-dependent Madelung term almost equals the crystal energy, dipole contributions of $1 / r^{2}$-dependency were found to account for only 5 to $10 \%$. Hence, higher multipoles will contribute even less. In order to clarify the range of validity of the point multipole model, further investigations of more heteropolar crystals under inclusion of higher electrostatic moments will be needed.

I would like to thank R. Rudert, Max-Planck-Institut für Kolloidund Grenzflächenforschung, D. Haase, Institut für Physikalische und Theoretische Chemie, I. Peschel, Institut für Theorie der kondensierten Materie, all in Berlin, and M. Schmitz, hydronic GmbH, Bitburg, for very helpful discussions and their support of this work.

\section{References}

1. Madelung, E.: Phys. ZS. XIX, 524 (1918)

2. Born, M.: Problems of atomic dynamics, p. 168-170. Cambridge: MIT Press 1926

3. Khan, M.A.: J. Phys. C9, 81 (1976)

4. Ashicroft, N.W., Mermin, N.D.: Solid State Physics, p. 407, 555. Tokyo: CBS Publishing Japan 1981

5. Kröger, F.A.: The chemistry of imperfect crystals, p. 249. Amsterdam: North-Holland 1974

6. O’Regan, B., Grätzel, M.: Nature 353, 737 (1991)
7. Ennaoui, A., Fiechter, S., Pettenkofer, Ch., Alonso-Vante, N., Büker, K., Bronold, M., Höpfner, Ch., Tributsch, H.: Sol. Energy Mater. Sol. Cells 29, 289 (1993)

8. Baur, W.H.: Acta Crystallogr. 14, 209 (1961)

9. Birkholz, M.: J. Phys.: Condens. Matter 4, 6227 (1992)

10. Kanamori, J., Moriya, T., Motizuki, K., Nagamiya, T.: J. Phys. Soc. Jap. 10, 93 (1955)

11. Nijboer, B.R.A., de Wette, F.W.: Physica 23, 309 (1957)

12. de Wette, F.W., Nijboer, B.R.A.: Physica 24, 1105 (1958)

13. de Wette, F.W.: Physica 25, 1225 (1959)

14. Bertaut, E.F.: J. Phys. (Paris) 39, 1331 (1978)

15. de Wette, F.W.: Phys. Rev, 123, 103 (1961)

16. Taylor, T.T.: Phys. Rev. 127, 120 (1962)

17. Hewitt, R.R., Taylor, T.T.: Phys. Rev. 125, 524 (1962)

18. Taylor, T.T., Das, T.P.: Phys. Rev. 133, A1327 (1964)

19. Sharma, R.R., Das, T.P.: J. Chem. Phys. 41, 3581 (1964)

20. Artmann, J.O.: Phys. Rev. 143, 541 (1966)

21. Artmann, J.O.: Phys. Rev. 173, 337 (1968)

22. Buckingham, A.D.: In: Intermolecular Forces, p. 107. Hirschfelder, J.O. (ed.). New York: Interscience 1967

23. Stone, A.J., Price, S.L.: J. Phys. Chem. 92, 3325 (1988)

24. Kitaigorodski, A.I.: Molekülkristalle. Berlin: Akademie-Verlag 1979

25. Metzger, R.M. (ed.): Crystal cohesion and conformational energies. Berlin: Springer 1981

26. Rozenbaum, V.M.: JETP Lett. 59, 173 (1994)

27. Birkholz, M.: Z. Phys. B 96, 333 (1995)

28. Jenkins, H.D.B.: In: CRC Handbook of chemistry and physics, p. D 100. Weast, R.C. (ed.). Boca Raton: CRC Press 1986

29. Jackson, J.D.: Classical electrodynamics, Chap. 4. New York: Wiley 1975

30. Bhagavantam, S., Suryanarayana, D.: Acta Crystallogr. 2, 21 (1949)

31. Rudert, R.: (Personal communication 1992)

32. Radzig, A.A., Smirnov, B.M.: Reference data on atoms, molecules and ions. Berlin: Springer 1985

33. Cotton, F.A., Wilkinson, G.: Advanced inorganic chemistry, p. 58. New York: Wiley 1972

34. Birkholz, M., Fiechter, S., Hartmann, A., Tributsch, H.: Phys. Rev. B 43, 11926 (1991)

35. Parker, R.A.: Phys. Rev. 124, 1713 (1961)

36. Kingsbury, P.I.: Acta Crystallogr. A 24, 578 (1968)

37. Jorgensen, J.D., Dabrowski, B., Shiyou Pei, Hinks, D.G., Soderholm, L., Morosin, B., Schirber, J.E., Venturini, E.L., Ginley, D.S.: Phys. Rev. B 38, 11337 (1988)

38. Birkholz, M., Rudert, R.: Z. Phys. B (in preparation)

39. Bednorz, J.G., Müller, K.A.: Z. Phys. B 64, 189 (1986)

40. Cava, R.J., Hewat, A.W., Hewat, E.A., Batlogg, B., Marezio, M., Rabe, K.M., Krajewski, J.J., Peck, W.F., Rupp, L.W.: Phys. C 165, 419 (1990)

41. Rudert, R., Birkholz, M.: ELC - A Computer Program for the Calculation of Electrostatic Lattice Coefficients 1994

42. Mahan, G.D., Subbaswamy, K.R.: Local Density Theory of Polarizability. New York: Plenum Press 1990 